\title{
Libration induced stretching mode excitation for pump-probe spectroscopy in pure liquid water
}

\author{
Wafa Amir, ${ }^{\text {a) }}$ Guilhem Gallot, ${ }^{\text {b) }}$ and François Hache \\ Laboratory for Optics and Biosciences, CNRS UMR 7645, INSERM U451, Ecole polytechnique, \\ 91128 Palaiseau, France
}

(Received 14 June 2004; accepted 4 August 2004)

\begin{abstract}
We developed an experimental approach to study pure liquid water in the infrared and avoid thermal effects. This technique is based on libration induced stretching excitation of water molecules. A direct correspondence between frequencies within the libration and $\mathrm{OH}$ stretching bands is demonstrated. Energy diffusion is studied in pure liquid water by measuring wave packet dynamics of $\mathrm{OH}$ stretching vibrator with infrared femtosecond spectroscopy. Wave packet dynamics reveals ultrafast energy dynamics and reflects $130 \mathrm{fs}$ intermolecular energy transfer between water vibrators. Energy diffusion is almost two orders of magnitude faster than self diffusion in water. (C) 2004 American Institute of Physics. [DOI: 10.1063/1.1800952]
\end{abstract}

\section{INTRODUCTION}

Liquid water plays a central role in chemistry and profoundly influences all molecular interactions in biological systems. The mechanisms of energy transfer in liquid water are essential for the understanding of a broad range of phenomena such as acid-base reaction, solvatation, ionic channels, or protein folding. For a decade, $\mathrm{H}_{2} \mathrm{O}$ molecule has been extensively studied by pump-probe techniques on a femtosecond time scale, often as an impurity in $\mathrm{D}_{2} \mathrm{O}$ $\left(\mathrm{HDO} / \mathrm{D}_{2} \mathrm{O}\right),{ }^{1-7}$ or in $\mathrm{CCl}_{4} \cdot{ }^{8}$ These experiments consist in following in time a vibrationally excited wave packet. Evolution of the amplitude and frequency of this wave packet yields information on the energy relaxation as well as on the hydrogen bonding network. Even though the geometrical properties of water (translation, rotation) are similar in these various configurations, pure water is nevertheless different from these systems as it is expected to exhibit resonant intermolecular energy transfer which precludes the local mode description utilized in previous works. These resonant effects are essential to fully understand water interaction with solute, in particular, with biological molecules such as proteins. However, very few experimental data are available on pure $\mathrm{H}_{2} \mathrm{O} .{ }^{9,10}$ The main experimental drawback is its very high infrared absorption, necessitating thin samples and leading to heavy thermal effects that confuse the interpretation. Here, we propose a femtosecond pump-probe technique on thick samples allowing direct observation of resonant dipolar energy transfer in pure water.

\section{HOT-BAND ABSORPTION}

The infrared spectrum of $\mathrm{H}_{2} \mathrm{O}$ (see Fig. 1) is dominated by a very strong absorption between 3000 and $3600 \mathrm{~cm}^{-1}$ due to symmetric and antisymmetric stretching modes $\nu_{O H}$.

\footnotetext{
${ }^{a)}$ Present address: Colorado School of Mines, 1500 Illinois St., Golden, CO 80401.

${ }^{b)}$ Author to whom correspondence should be addressed. Electronic mail: Guilhem.Gallot@polytechnique.fr
}

A direct excitation in this region implies performing the experiments on very thin samples and induces problematic thermal effects. However, the spectrum also shows an interesting combination band around $2800 \mathrm{~cm}^{-1}$, involving transitions from libration $\nu_{L}$ to stretching modes $\nu_{O H} \cdot{ }^{11}$ Low frequency modes are dominated by translational modes (hydrogen bond stretching) below $200 \mathrm{~cm}^{-1}$ and libration modes (hindered rotations) around $700 \mathrm{~cm}^{-1}$. The transition $\nu_{L} \rightarrow \nu_{O H}$ corresponds to a transition from a libration "hot band" to the stretching band. Therefore it only involves water molecules which are in an excited libration mode. The probability to find a molecule in such an excited state decreases with energy according to Boltzmann distribution $p$ $\propto \exp (-E / k T)$. At room temperature, this probability is a few percent, implying that the $\nu_{L} \rightarrow \nu_{O H}$ transition is allowed for a restricted number of water molecules. The idea is to excite the stretching $\mathrm{OH}$ vibrators by a pump pulse tuned at the difference of frequencies between libration and stretching modes. As a result, the libration mode induced absorption "dilutes" the pump excitation like in $\mathrm{HDO} / \mathrm{D}_{2} \mathrm{O}$ systems, but conserves the geometrical and resonant characteristics of pure water. The evolution of the excited molecules is then monitored by probing the $\left(\nu_{O H}=1 \rightarrow \nu_{O H}=2\right)$ transition whose cross section is much stronger than the $\left(\nu_{L}=1\right.$ $\left.\rightarrow \nu_{O H}=1\right)$ transition.

A fundamental issue for studying pure water dynamics is the possibility to select a subset of water molecules as this is currently done in $\mathrm{HDO} / \mathrm{D}_{2} \mathrm{O}$ systems. In order to achieve such a selection, the hot-band transition frequency $\nu_{L}$ $\rightarrow \nu_{O H}$ must display a one to one correspondence with the stretching frequency $\nu_{O H}$. No strong structure is observed near $2800 \mathrm{~cm}^{-1}$ in the linear spectrum of water because the band is wide and relatively close to the stretching modes. Then further modelization and analyzation are required to justify this statement. We want now to address this question by investigating more carefully the libration modes of water molecules. Let us first consider the libration modes and their interaction with the environment. This coupling has been 


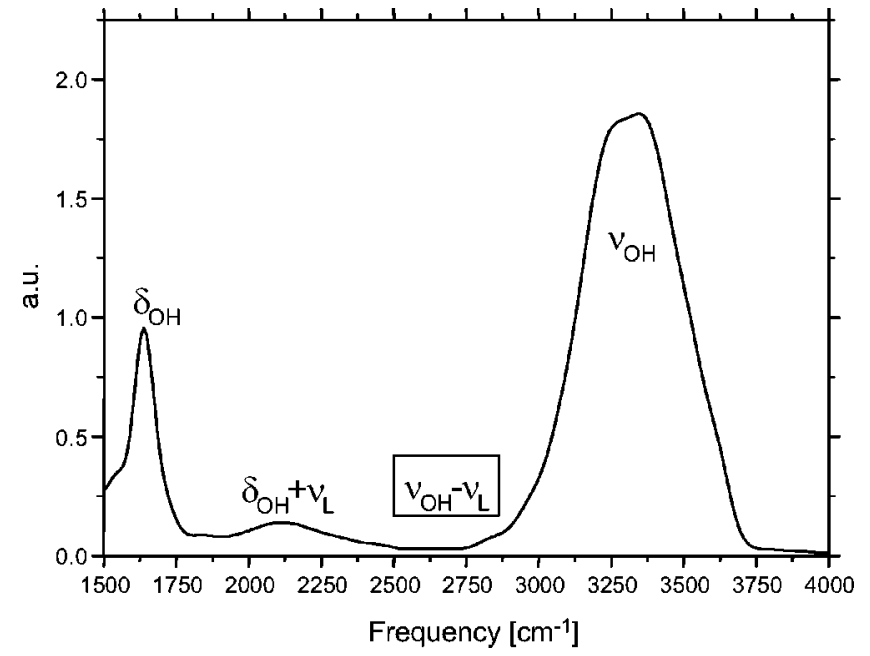

FIG. 1. The linear absorption spectrum of $\mathrm{H}_{2} \mathrm{O}$ in the midinfrared is dominated by three peaks: the symmetric and antisymmetric stretching modes $\nu_{O H}$ around $3300 \mathrm{~cm}^{-1}$, the bending mode $\delta_{O H}$ at $1640 \mathrm{~cm}^{-1}$ and a combination band between the bending and libration modes $\left(\delta_{O H}+\nu_{L}\right)$ around $2200 \mathrm{~cm}^{-1}$. Furthermore on the red side of $\nu_{O H}$, a combination band between $\nu_{O H}$ and libration modes extend from 2300 to $2900 \mathrm{~cm}^{-1}$.

studied in detail by Gaiduk and Gaiduk. ${ }^{12}$ The model is the following. The libration bands are due to reorientation of the dipole moment of the water molecule as a whole in the hydrogen bonding network [Fig. 2(a)]. The water molecule moves in a rectangular potential profile with the depth $U_{0}$ and angular width $2 \beta$ [Fig. 2(b)]. The reorientation is described by the hindered rotation angle $\beta$, which accounts for the interaction with the surrounding molecules and then depends on the hydrogen bonding density that hinders the molecular rotation. Molecules with energy $H<U_{0}$ perform libration, and molecules with $H>U_{0}$ perform free rotation. The libration frequency $\nu_{L}$ is related to $\beta$ by ${ }^{12}$

$$
\nu_{L}=\frac{1}{4 c \beta} \sqrt{\frac{3 k_{B} T}{I}}
$$

where $c$ is the light velocity, $k_{B}$ the Boltzmann constant, $T$ the temperature, and $I=1.483 \times 10^{-40} \mathrm{~g} \mathrm{~cm}^{2}$ the inertial moment of the molecule. The angle $\beta$ relies on the molecular density by, ${ }^{13}$

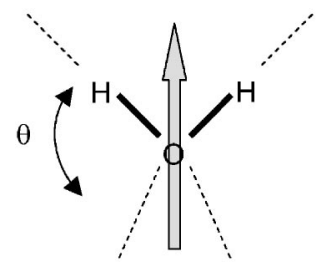

(a)

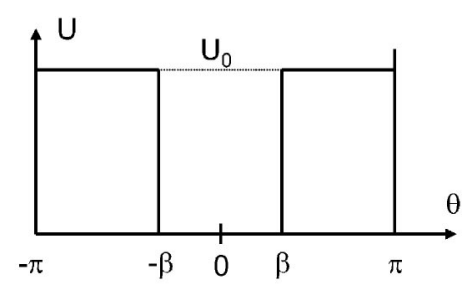

(b)
FIG. 2. Libration model for liquid water (from (Ref. 12). (a) A water molecule is linked to four neighbors by hydrogen bonding (doted lines) and oscillates in the hydrogen bonding network with an angular amplitude $2 \beta$. (b) The molecule evolves in a rectangular potential profile, with an angular width $2 \beta$ and depth $U_{0}$.

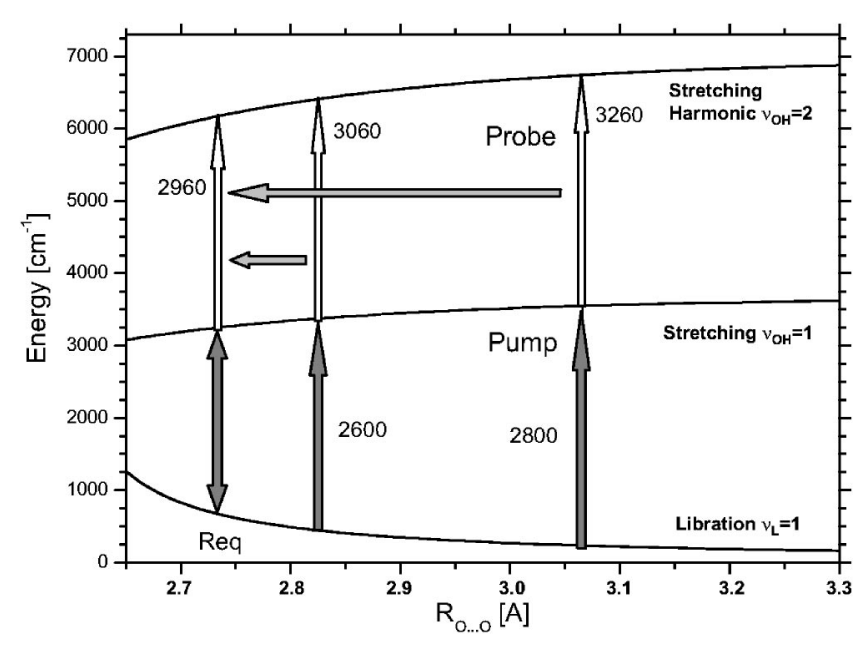

FIG. 3. Mode levels involved in the pump-probe scheme. The pump populates the first stretching mode from the first exited libration mode $\left(\nu_{L}=1\right.$ $\rightarrow \nu_{O H}=1$ ). A pump at $2800 \mathrm{~cm}^{-1}$ (respectively, $2600 \mathrm{~cm}^{-1}$ ) excites the first stretching mode at an energy of about $3550 \mathrm{~cm}^{-1}$ (respectively, $3380 \mathrm{~cm}^{-1}$ ). Due to cross section consideration, the probe reads the second transition $\left(\nu_{O H}=1 \rightarrow \nu_{O H}=2\right)$ starting at $3260 \mathrm{~cm}^{-1}$ (respectively, $3060 \mathrm{~cm}^{-1}$ ). After excitation, the wave packets evolve in both cases back to thermodynamical equilibrium at $3250 \mathrm{~cm}^{-1}$ which the probe reads at $2960 \mathrm{~cm}^{-1}$.

$$
\beta=\frac{\pi}{8} \sqrt{\frac{m_{H} M}{I}}\left[\left(\frac{M}{N_{A} \rho(T)}\right)^{1 / 3}-\sigma\right],
$$

where $m_{H}$ is the proton mass, $M$ the molecular weight, $N_{A}$ the Avogadro's number, $\rho$ the density, and $\sigma=2.9 \mathrm{~A}$ the effective diameter of the molecule. The libration frequency $\nu_{L}$ is therefore directly correlated to the average nearby hydrogen bonding length $R_{O \cdots O}$ : a dense (loose) hydrogen bonding network is then responsible for high (low) frequency libration modes. The density is related to the average hydrogen bonding distance $R_{O \cdots O}$ by $\rho=C / R_{O O}^{3}$. At thermodynamical equilibrium, $C=2.035 \times 10^{-23} \mathrm{~g}$. Equation (1) then becomes

$$
\nu_{L}\left[\mathrm{~cm}^{-1}\right]=\frac{136.3}{1.135 R_{O O}[A]-2.9} .
$$

Furthermore, it is well known that the average stretching vibration frequency $\nu_{O H}$ is also correlated to $R_{O \cdots O} \cdot{ }^{14}$ Even though a dispersion exists in the relationship between $\nu_{O H}$ and $R_{O \cdots O},{ }^{15,16}$ the pump induced wave packet can be described by the average value of this dispersive relationship. Therefore, to a given hydrogen bonding network corresponds an average libration and stretching frequency. In order to address this point more quantitatively, we have carried out quantum mechanical calculation of the first and second stretching mode frequencies by solving a one-dimensional Schrödinger equation for the O-H coordinate $R_{O H}$ using an anharmonic Lippincott-Schröder potential. ${ }^{17}$ This calculation shows that both $\left(\nu_{O H}=0 \rightarrow \nu_{O H}=1\right)$ and $\left(\nu_{O H}=1 \rightarrow \nu_{O H}\right.$ 2) transition frequencies increase when $R_{O \cdots O}$ increases. The structure of the librational and vibrational levels, as depicted in Fig. 3, allows one to understand that $\nu_{P u m p}=\nu_{O H}$ $-\nu_{L}$ displays a one to one correspondence with $\nu_{O H}$ and 
therefore allows selective excitation of a stretching mode wave packet. The experimental principle is depicted in Fig. 3. At thermodynamical equilibrium, $\nu_{L}=720 \mathrm{~cm}^{-1}$ and $\nu_{O H}=3250 \mathrm{~cm}^{-1}$ corresponding to a transition frequency $\nu_{L} \rightarrow \nu_{O H}=2530 \mathrm{~cm}^{-1}$. An intense laser pulse tuned above the latter frequency creates an excited wave packet which will evolve back to the equilibrium position, revealing the dynamics of liquid water, as observed in Fig. 3. For instance, a pump pulse tuned at $2800(2600) \mathrm{cm}^{-1}$ creates an excited wave packet $270(70) \mathrm{cm}^{-1}$ above the equilibrium frequency.

The question of probing this excited wave packet must now be addressed carefully because a four-level quantum system is implied. From the first excited state $\nu_{O H}=1$, three transitions are available: to the ground state $\left(\nu_{O H}=1\right.$ $\rightarrow \nu_{O H}=0$, bleaching), to the second excited state $\left(\nu_{O H}=1\right.$ $\rightarrow \nu_{O H}=2$, induced absorption) or back to the libration state $\left(\nu_{O H}=1 \rightarrow \nu_{L}\right)$. The resonance of $\nu_{O H}$ mode is $3250 \mathrm{~cm}^{-1}$, with an anharmonicity of $300 \mathrm{~cm}^{-1}$ toward the lower frequencies. Thus, the effect of bleaching of the transition is totally negligible compared to induced absorption in the $2600-3000 \mathrm{~cm}^{-1}$ range which is covered by our probe. The contribution of transition back to the libration state may be estimated from the $\nu_{O H}=1 \rightarrow \nu_{L}$ transition cross section. From the linear absorption spectrum and taking into account the reduced population of the libration modes, this latter is found to be at least four times weaker than the induced absorption one in the $2600-3000 \mathrm{~cm}^{-1}$ range. The $\nu_{O H}=1$ $\rightarrow \nu_{L}$ transition should therefore scarcely contribute to the pump-probe signal. In conclusion, one expects the pumpprobe differential signal to be strongly dominated by the $\nu_{O H}=1 \rightarrow \nu_{O H}=2$ transition, implying an increase of the absorption of the probe induced by the pump.

\section{EXPERIMENTAL SETUP}

To perform pump-probe hole burning experiments in the midinfrared range, energetic laser pulses are required. The central element of the infrared laser source ${ }^{18}$ is a titaniumsapphire amplifier, delivering $130 \mathrm{fs}$ pulses at $800 \mathrm{~nm}$ with a repetition rate of $1 \mathrm{kHz}$. It drives two lines of pulses independently tunable in the midinfrared. The principle of the generation is the parametric amplification of a quasicontinuum in the near infrared, followed by a frequency mixing in the midinfrared. The features of the sources are as follows. The more energetic line (the pump) has a duration of $150 \mathrm{fs}$ and a spectral width of $65 \mathrm{~cm}^{-1}$. It delivers more than $10 \mu \mathrm{J}$ between 2800 and $3800 \mathrm{~cm}^{-1}$. The independently tunable weaker line (the probe) has similar characteristics but with a maximum energy ten times less. The delay between the pump and the probe is precisely controlled by a computer. The sample cell is $40 \mu \mathrm{m}$ thick and contains pure water at room temperature. The sample is circulated to avoid heating problems. The pump and the probe beams are focused in the sample by two $25 \mathrm{~mm}$-focal length calcium fluoride lenses. The angle between the pump and the probe beams is $15^{\circ}$. In order to reduce the noise perturbation, the probe signal is normalized by the signal issued from a small part of the probe beam reflected before the cell. Furthermore, the pump beam is chopped at half the repetition rate of the

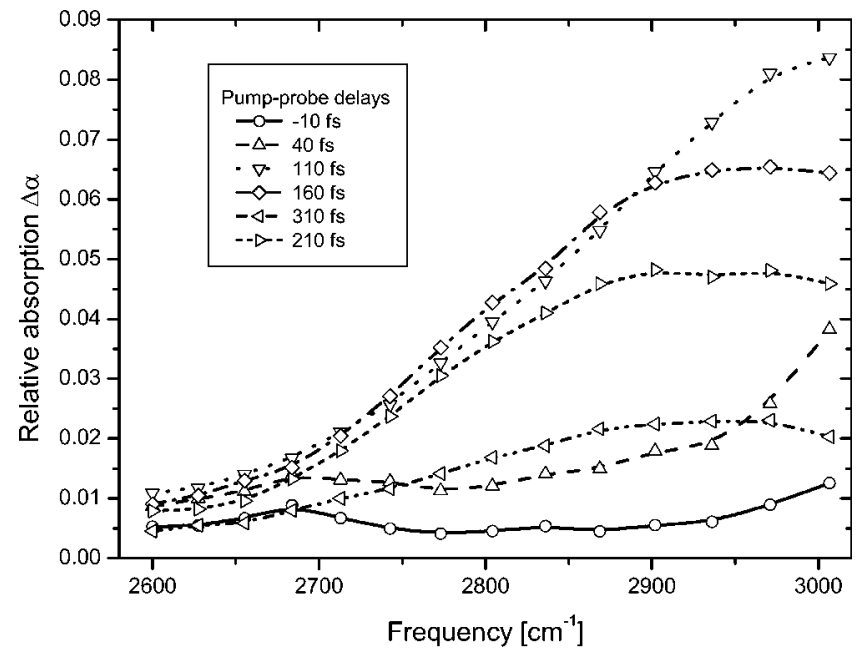

FIG. 4. Differential spectra in pure water with a pump tuned at $2800 \mathrm{~cm}^{-1}$ and six pump-probe delays. The sample cell is $40 \mu \mathrm{m}$ thick.

laser (i.e. $500 \mathrm{~Hz}$ ), in order to obtain successively a probe signal with and without the pump. With this procedure, we achieve signal to noise ratio of $10^{4}$. For a given pump frequency, differential spectra are recorded by tuning the probe frequency for several pump-probe delays.

\section{EXPERIMENTAL DATA}

We now present differential spectra, that is, probe absorption with the pump minus probe absorption without the pump. The pump frequency is tuned at $2800 \mathrm{~cm}^{-1}$ and the probe frequencies cover the range $2600-3000 \mathrm{~cm}^{-1}$. The frequencies above $3000 \mathrm{~cm}^{-1}$ are experimentally unobservable because of high absorption of water. The differential spectra are presented in Fig. 4. First, one notices an overall increase of absorption in presence of the pump, showing that induced absorption from first to second stretching excited state $\left(\nu_{O H}=1 \rightarrow 2\right)$ is the dominant contribution for the differential spectra. Second, short delay spectra ( -10 and $40 \mathrm{fs}$ delays) show no evidence for spectral substructure around $2800 \mathrm{~cm}^{-1}$, indicating that $\nu_{O H}=1 \rightarrow \nu_{L}$ transition is totally negligible. As expected, these differential spectra are the signature of the wave packet evolution of the $\nu_{O H}=1$ state, probed through $\nu_{O H}=1 \rightarrow 2$ transition, because other transitions $\left(\nu_{O H}=1 \rightarrow \nu_{L}\right.$ and $\left.\nu_{O H}=1 \rightarrow 2\right)$ are negligible. The time evolution of the spectrum shape is related to energetic reorganization of the wave packet under the influence of other water molecules and hydrogen bonding network. More precisely, in Fig. 4, for short delays, the spectra exhibit a maximum beyond $3000 \mathrm{~cm}^{-1}$ which will be clarified further. This maximum clearly moves toward lower frequencies and stabilizes around $2900 \mathrm{~cm}^{-1}$ after a delay of $200 \mathrm{fs}$. Simultaneously, the energy integral of the spectra decreases very rapidly with a dynamics of less than 200 fs. The energy is maximum for a delay of about $100 \mathrm{fs}$, and has almost completely relaxed $200 \mathrm{fs}$ later. This shows a very fast dynamics of the energy stored in the excited wave packet, as well as the energetic reorganization of the wave packet. Both exhibit characteristic times less than $200 \mathrm{fs}$. 


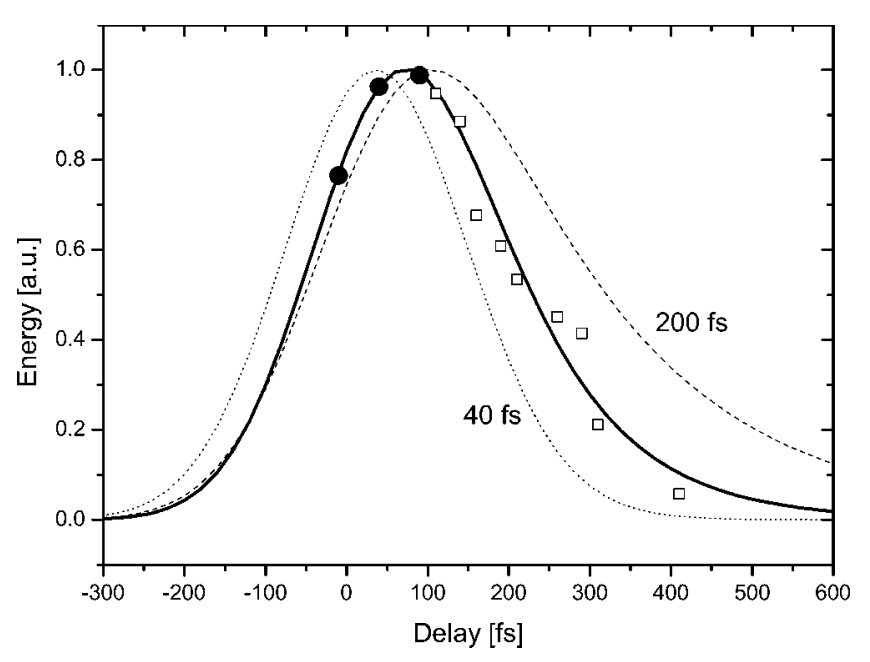

FIG. 5. Energy relaxation of the wave packet. The hollow squares are experimental data and the lines stand for theoretical curves (Ref. 19) for three relaxation times: solid (110 fs), dashed (200 fs), and dotted (40 fs). The solid circles are extrapolated energy values for a relaxation time of $110 \mathrm{fs}$.

More precise information may be extracted from the data. Due to incomplete knowledge of the differential spectra at short delays, Gaussian fit is not directly applicable. Indeed, a far and energetic wave packet will have roughly the same truncated spectral shape as a less energetic but closer wave packet. However, at longer delays, the shape of the wave packet allows us to extract energy and position with precision. From the knowledge of energetic evolution at delays longer than $100 \mathrm{fs}$, deduction of short delays dynamics is then possible. Evolution of the wave packet energy has been calculated ${ }^{19}$ and our data yields a dynamics of 110 $\pm 20 \mathrm{fs}$, from which short time dynamics is deduced (see Fig. 5). This dynamics is very fast, even shorter than the pulse width. However it is well established than sub-pulsewidth temporal resolution can be achieved providing a very precise knowledge of the pump-probe delay and the study of the signal shape. The first point is made possible by precisely synchronizing the pump and the probe in a GaSe nonlinear crystal for each couple of pump and probe frequencies to avoid small fluctuation of delays during the probe tuning. From the pump-probe correlation measurement, one can estimate the delay precision to be less than 20 fs. The second point is clearly demonstrated in Fig. 5 by fits of the energy dynamics with characteristic times shorter (40 fs) and longer (200 fs), which can be discriminated from best fit, and provides the estimation for the fitting precision. The last point is the absence of optical coherent effects in the experiment, as pointed out by several other experiments on water. ${ }^{1-7}$ With the calculated energy of the wave packet at short delays, Gaussian fit provides the position of the wave packet with good precision. The result is depicted in Fig. 6. As expected, the central position of the wave packet evolves from higher to lower frequencies. The amplitude of the displacement is important: about $220 \mathrm{~cm}^{-1}$. Indeed, the wave packet moves on more than half of the width of the $\mathrm{OH}$ vibrator. At short delays, the wave packet position moves slightly from the starting point around $3180 \mathrm{~cm}^{-1}$, because the pump is still generating the excited wave packet. Once the pump disap-

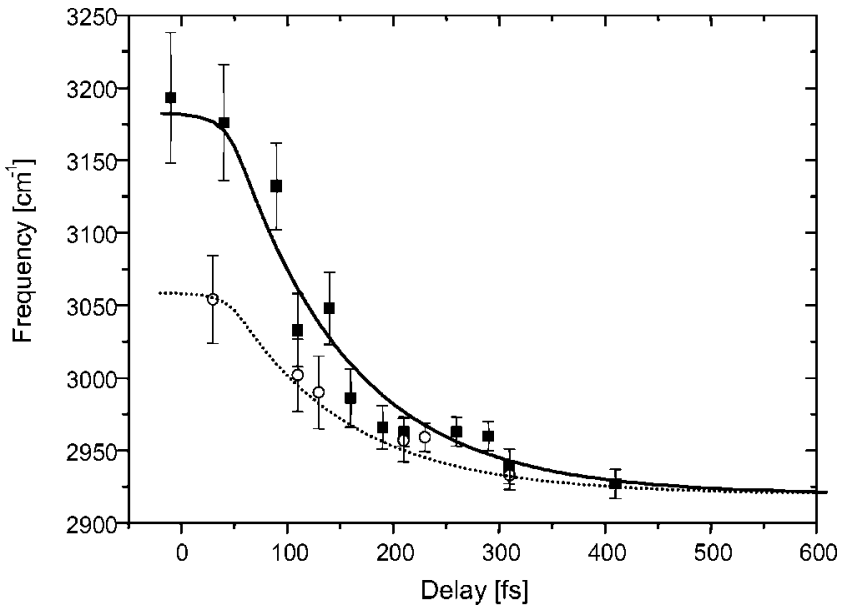

FIG. 6. Evolution of the central position of excited wave packets in pure water. The pump creates an excited wave packet, the position of which quickly evolves toward the equilibrium position, with a characteristic time of $110 \mathrm{fs}$. Solid squares (respectively, hollow circles) are experimental positions corresponding to a pump frequency of 2800 (respectively, 2600) $\mathrm{cm}^{-1}$. Solid lines are theoretical curves from Ref. 19.

pears, the wave packet evolves surprisingly quickly, being back to an equilibrium position in less than $300 \mathrm{fs}$. The origin around $3180 \mathrm{~cm}^{-1}$ is associated with the probing of the $\nu_{O H}=1 \rightarrow 2$ transition, corresponding to a $\nu_{O H}=0 \rightarrow 1$ transition of $3495 \mathrm{~cm}^{-1}$. It should be noted that error bars are relatively large at short delays. It comes from the uncertainty of the fitting procedure due to incomplete spectral bands. However, it does not question the observation of spectral diffusion in pure water because the fits are based on precise wing shapes and energy knowledge. An other set of experiments have been performed with the pump frequency tuned at $2600 \mathrm{~cm}^{-1}$, that is, to say closer from thermodynamical equilibrium position. As expected, one observes (see Fig. 6) a spectral displacement similar to the one obtained with the pump at $2800 \mathrm{~cm}^{-1}$. The starting point of the wave packet is lower, around $3060 \mathrm{~cm}^{-1}$ and the amplitude is less important: $100 \mathrm{~cm}^{-1}$. The equilibrium position is once again at $2920 \mathrm{~cm}^{-1}$. Excitation at $2800 \mathrm{~cm}^{-1}$ (respectively $2600 \mathrm{~cm}^{-1}$ ) provides a wave packet centered around $3180 \mathrm{~cm}^{-1}$ (respectively $3060 \mathrm{~cm}^{-1}$ ), in good agreement with our model, and shows the nature of the librationstretching coupling. A pump at higher frequency selects a subset of water molecules with larger average hydrogen bond length (see Fig. 3) due to both the increase of the stretching frequency and decrease of hot libration frequency with respect to $R_{O \cdots O}$. This also explains why moving the pump frequency $200 \mathrm{~cm}^{-1}$ only moves the wave packet $120 \mathrm{~cm}^{-1}$, in agreement with the model.

\section{MODEL AND DISCUSSION}

To extract quantitative information from the experimental data, we computed the behavior of the wave packet in spectral diffusion regime ${ }^{19}$ which requires the spectral diffusion characteristic time $\tau_{\text {diff }}$ as only dynamical parameter besides the pump and probe pulse duration. The best agreement, shown in Fig. 6, has been obtained with a characteristic time $\tau_{\text {diff }}$ equal to $110 \pm 15$ fs. It should be noted that the 
parameters used to fit the wave packet displacements for the two pump frequencies at 2800 and $2600 \mathrm{~cm}^{-1}$ are the same, except of course for the amplitude of the displacement. The dynamics of the wave packet is once again shorter than the pulse duration. However the high precision obtained comes from the taking into account of the whole spectrum. Thus small spectral displacement and fast dynamics are easily discernible.

The physical origin of this spectral displacement may now be discussed. By analogy with solvatochromism found in $\mathrm{HDO} / \mathrm{D}_{2} \mathrm{O},{ }^{1}$ the first hypothesis would be to attribute this displacement to hydrogen bonding reorganization. The associated dynamics implies two time scales ${ }^{20}$ with approximatively the same amplitude: a long time of about $700 \mathrm{fs}$ (Ref. $1,3)$ for hydrogen bonding network reorganization, and a short time of less than 100 fs related to the coherence loss of the vibrator through a single hydrogen bonding ${ }^{5}$ and which requires photon-echo type measurements. ${ }^{5,716}$ The measured time scale of $110 \mathrm{fs}$ is far too short to support this hypothesis as hydrogen bonding network reorganization in pure water is very similar to the one in $\mathrm{HDO} / \mathrm{D}_{2} \mathrm{O} .{ }^{21}$ Furthermore, our experiment is very similar to previous ones on $\mathrm{HDO} / \mathrm{D}_{2} \mathrm{O}$, except of course the excitation principle. If this hypothesis was valid, we should have obtained the long time component due to network reorganization which is not the case. Another possibility might be related to intermolecular dynamics between $\nu_{O H}$ and $2 \delta$ modes. However Raman experiments ${ }^{22}$ showed no evidence at all for such a transfer, and the spectral separation between the two modes seems too large to support a vibrational transfer of $110 \mathrm{fs}^{22}$ Vibrational Stokes shift can neither explain our results. It has been found equal to $70 \mathrm{~cm}^{-1,3}$ but its amplitude is too small compared to $220 \mathrm{~cm}^{-1}$. Furthermore, it is independent on the excitation frequency, thus pumping at 2600 and $2800 \mathrm{~cm}^{-1}$ would lead to redshifts of equal amplitude, in contradiction with experimental results. Actually, the main difference between pure water and $\mathrm{HDO} / \mathrm{D}_{2} \mathrm{O}$ is that in pure water, a water molecule is surrounded by about four other similar water molecules. Vibrational energy transfer (VET) has therefore to be taken into consideration. Through VET, vibration from an excited water molecule diffuses to neighboring molecules, quickly altering the environment of the probed vibrator. Such VET has already been observed by Woutersen and Bakker. ${ }^{23}$ Poulsen et $_{\text {al. }}{ }^{24}$ simulated VET in pure water, and found a time transfer from 80 to $250 \mathrm{fs}$, in very good agreement with our data. This allows us to depict the model of diffusion in pure water as follows: the pump pulse, via libration modes, excites $\nu_{O H}$ vibrator at a given frequency. Each excited molecule is linked to four other water molecules by hydrogen bonding, which is a very good vibrational energy transfer vector. Neighboring vibrators are then progressively perturbed by successive hopping of energy. Their environment being different from the initial vibrator, their frequencies are changed accordingly. From vibrators excited above the equilibrium frequency as in our experiments, the wave packet frequency moves back to equilibrium position with very high speed, much more rapidly than the hydrogen bonding network reorganization. From this hopping diffusion process, one can attempt to access the energy diffusion coefficient in pure water. It depends on the number of hops required to investigate the whole geometrical conformations and is directly linked to the loss of geometrical correlation between water molecules. Radial distribution $g_{O O}$ in water ${ }^{25}$ shows that correlation is lost after the second shell surrounding the reference molecule. From that we estimate the hopping distance to be the mean $\mathrm{OO}$ distance between water molecules $l=0.285 \mathrm{~nm}$, with a hopping time equivalent to the measured diffusion time, $\tau=100 \mathrm{fs}$. Einstein's relationship $D$ $=l^{2} / 6 \tau$ gives a diffusion constant $D=1.410^{-3} \mathrm{~cm}^{2} / \mathrm{s}$, which is 60 times faster than self-diffusion coefficient in water. $^{26}$

\section{CONCLUSION}

This study shows that the response of pure water to an environmental change is strongly influenced by ultrafast energy transfer, with a characteristic time of about 100 fs. Resonant intermolecular energy transfer between water molecules seems to be the dominating process, even though intramolecular energy transfer between symmetric and asymmetric stretching modes could play a role. Resonant energy transfer in pure water needs to be taken into account in numerical simulations, such as proton transfer ${ }^{27,28}$ and waterprotein interaction. ${ }^{29}$ Further theoretical models and numerical simulations of pure water are required to get a precise picture of the multiple modes found in pure water.

\section{ACKNOWLEDGMENTS}

The authors would like to thank S. Bratos, J.-C. Leicknam, R. Vuilleumier and S. Pommeret for helpful discussion.

${ }^{1}$ G. M. Gale, G. Gallot, F. Hache, N. Lascoux, S. Bratos, and J.-C. Leicknam, Phys. Rev. Lett. 82, 1068 (1999).

${ }^{2}$ R. Laenen, C. Rauscher, and A. Laubereau, Phys. Rev. Lett. 80, 2622 (1998)

${ }^{3}$ S. Woutersen and H. J. Bakker, Phys. Rev. Lett. 83, 2077 (1999).

${ }^{4}$ J. Stenger, D. Madsen, P. Hamm, E. T. J. Nibbering, and T. Elsaesser, Phys. Rev. Lett. 87, 027401 (2001).

${ }^{5}$ S. Yeremenko, M. S. Pshenichnikov, and D. A. Wiersma, Chem. Phys. Lett. 369, 107 (2003).

${ }^{6}$ C. P. Lawrence and J. L. Skinner, Chem. Phys. Lett. 369, 472 (2003).

${ }^{7}$ C. J. Fecko, J. D. Evaes, J. J. Loparo, A. Tokmakoff, and P. L. Geissler, Science 301, 1698 (2003)

${ }^{8}$ H. Graener and F. Seifert, J. Chem. Phys. 98, 36 (1993).

${ }^{9}$ A. Pakoulev, Z. Wang, and D. D. Dlott, Chem. Phys. Lett. 371, 594 (2003).

${ }^{10}$ A. J. Lock and H. J. Bakker, J. Chem. Phys. 117, 1708 (2002).

${ }^{11}$ J.-J. Max and C. Chapados, J. Chem. Phys. 116, 4626 (2002).

${ }^{12}$ V. I. Gaiduk and V. V. Gaiduk, Mendeleev Commun. 2, 76 (1997).

${ }^{13}$ V. I. Gaiduk and B. M. Tseitlin, Adv. Chem. Phys. 87, 125 (1994).

${ }^{14}$ W. Mikenda, J. Mol. Struct. 147, 1 (1986).

${ }^{15}$ C. P. Lawrence and J. L. Skinner, J. Chem. Phys. 118, 264 (2003).

${ }^{16}$ R. Rey, K. B. Møller, and J. T. Hynes, J. Phys. Chem. A 106, 11993 (2002).

${ }^{17}$ H. J. Bakker, H.-K. Nienhuys, G. Gallot, N. Lascoux, G. M. Gale, J.-C. Leicknam, and S. Bratos, J. Chem. Phys. 116, 2592 (2002).

${ }^{18}$ G. M. Gale, G. Gallot, F. Hache, and R. Sander, Opt. Lett. 22, 1253 (1997).

${ }^{19}$ S. Bratos and J.-C. Leicknam, J. Chem. Phys. 101, 4536 (1994).

${ }^{20}$ M. Diraison, Y. Guissani, J.-C. Leicknam, and S. Bratos, Chem. Phys. Lett. 258, 348 (1996).

${ }^{21}$ F. W. Starr, J. K. Nielsen, and H. E. Stanley, Phys. Rev. Lett. 82, 2294 (1999).

${ }^{22}$ A. Pakoulev, A. Wang, Y. Pang, and D. D. Dlott, Chem. Phys. Lett. 380, 404 (2003). 
${ }^{23}$ S. Woutersen and H. J. Bakker, Nature (London) 402, 507 (1999).

${ }^{24}$ J. A. Poulsen, G. Nyman, and S. Nordholm, J. Phys. Chem. A 107, 8420 (2003).

${ }^{25}$ A. Soper and M. G. Phillips, Chem. Phys. 107, 47 (1986).

${ }^{26}$ H. Weingartner, Z. Phys. Chem. (Leipzig) 132, 129 (1982).
${ }^{27}$ J. T. Hynes, Nature (London) 397, 565 (1999).

${ }^{28}$ D. Marx, M. E. Tuckerman, J. Hutter, and M. Parrinello, Nature (London) 397, 601 (1999).

${ }^{29}$ S. K. Pal, L. Zhao, and A. H. Zewail, Proc. Natl. Acad. Sci. U.S.A. 100, 8113 (2003) 